\title{
Impact of an enhanced recovery after surgery pathway on thoracoscopic lobectomy outcomes in non-small cell lung cancer patients: a propensity score-matched study
}

\author{
Céline Forster ${ }^{1}$, Valérie Doucet ${ }^{1}$, Jean Yannis Perentes ${ }^{1,2}$, Etienne Abdelnour-Berchtold ${ }^{1}$, Matthieu Zellweger ${ }^{1}$, \\ Mohamed Faouzi ${ }^{3}$, Hasna Bouchaab ${ }^{4}$, Solange Peters ${ }^{2,4}$, Carlo Marcucci ${ }^{2,5}$, Thorsten Krueger ${ }^{1,2}$, \\ Lorenzo Rosner ${ }^{5}$, Michel Gonzalez ${ }^{1,2}$
}

${ }^{1}$ Service of Thoracic Surgery, University Hospital of Lausanne (CHUV), Lausanne, Switzerland; ${ }^{2}$ University of Lausanne, Lausanne, Switzerland; ${ }^{3}$ Division of Biostatistics, Center for Primary Care and Public Health (Unisanté), University of Lausanne, Lausanne, Switzerland; ${ }^{4}$ Service of Medical Oncology, University Hospital of Lausanne (CHUV), Lausanne, Switzerland; '5ervice of Anesthesiology, University Hospital of Lausanne (CHUV), Lausanne, Switzerland

Contributions: (I) Conception and design: M Gonzalez, C Forster; (II) Administrative support: M Gonzalez; (III) Provision of study materials or patients: M Gonzalez, C Forster; (IV) Collection and assembly of data: M Gonzalez, C Forster; (V) Data analysis and interpretation: M Gonzalez, C Forster; (VI) Manuscript writing: All authors; (VII) Final approval of manuscript: All authors.

Correspondence to: Michel Gonzalez, MD. Service of Thoracic Surgery, Lausanne University Hospital (Centre Hospitalier Universitaire Vaudois), 1011 Lausanne, Switzerland. Email: michel.gonzalez@chuv.ch.

Background: This study evaluates the effect of enhanced recovery after surgery (ERAS) pathways on postoperative outcomes of non-small cell lung cancer (NSCLC) patients undergoing video-assisted thoracic surgery (VATS) lobectomy.

Methods: We retrospectively reviewed all consecutive patients undergoing VATS lobectomy for NSCLC between January 2014 and October 2019 and assigned them to the relevant group ("pre-ERAS" or "ERAS"). Length of stay, readmissions and complications within 30 days were compared between both groups. A propensity score-matched analysis was performed based on sex, age, type of operation, comorbidities, American Society of Anesthesiologists (ASA) score and preoperative pulmonary functions.

Results: A total of 307 records (164 male/143 female; 140 ERAS/167 pre-ERAS; median age: 67) were reviewed. There was no statistical difference in patient's characteristics. Overall ERAS compliance was 81\%. The ERAS group presented significantly shorter length of stay (median 5 vs. 7 days; $\mathrm{P}=0.004)$ without significant difference in cardiopulmonary complication rate $(27.1 \%$ vs. $35.9 \% ; \mathrm{P}=0.1)$. Readmission $(3.6 \%$ vs. $5.4 \% ; \mathrm{P}=0.75$ ) and duration of drainage (median 2 vs. 3 days; $\mathrm{P}=0.14$ ) were similar between groups. The propensity score-matched analysis showed that the length of hospital stay was reduced by 1.4 days $(\mathrm{P}=0.034)$ and the postoperative cardiopulmonary complication rate by $13 \%(\mathrm{P}=0.044)$ in the ERAS group.

Conclusions: Adoption of an ERAS pathway for VATS lobectomies in NSCLC patients has decreased the length of hospital stay and the cardiopulmonary complication rate without affecting the readmission rate.

Keywords: Video-assisted thoracic surgery (VATS); thoracoscopy; lobectomy; non-small cell lung cancer (NSCLC); enhanced recovery after surgery (ERAS)

Submitted Jul 06, 2020. Accepted for publication Nov 27, 2020.

doi: $10.21037 /$ tlcr-20-891

View this article at: http://dx.doi.org/10.21037/tlcr-20-891 


\section{Introduction}

Worldwide, lung cancer remains the leading cause of cancer-related deaths in men and women (1). Surgical resection is proposed in case of early stage non-small cell lung cancer (NSCLC). For stage I and II NSCLC, lobectomy with mediastinal lymph node dissection by video-assisted thoracic surgery (VATS) is the recommended treatment (2). Since its introduction in the 1990s, the VATS approach has shown many advantages over open thoracotomy, such as lower postoperative complication rate, shorter durations of hospital stay and drainage as well as reduction of postoperative pain (3-6).

Enhanced recovery after surgery (ERAS) programs were first introduced in colorectal surgery and have shown to reduce the length of hospital stay, the rate of postoperative complications and the overall costs (7-9). Since then, many other surgical specialties followed the trend with development of international ERAS guidelines (10-12). In thoracic surgery, several series demonstrated the positive impacts of ERAS programs, such as a reduction in the postoperative complication rate, the length of hospital stay and the costs (13-17). However, most series studying ERAS programs included heterogeneous and imbalanced patients' populations, undifferentiated surgical approaches and variable extent of pulmonary resections. Studies evaluating matched subgroups, such as cancer patients undergoing VATS lobectomy have not been reported and are needed to formally such programs.

The aim of our study was to evaluate the effects of the introduction of an ERAS pathway in NSCLC patients undergoing VATS lobectomy and to compare postoperative outcomes between pre-ERAS and ERAS groups using a propensity score-matched analysis.

The authors present the following article in accordance with the STROBE reporting checklist (available at http:// dx.doi.org/10.21037/tlcr-20-891).

\section{Methods}

\section{Patient selection and study design}

This retrospective study reviewed all adult patients (age $\geq 18$ years) with NSCLC of any stage who underwent a VATS lobectomy between January 2014 and October 2019 in our institution. The exclusion criteria were: intra-operative conversion to thoracotomy, patients presenting metastatic lesions and emergency procedures. The patients were then divided into two groups, according to their clinical management ("pre-ERAS" and "ERAS"), mainly defined by the timing of their operation before or after systematic introduction of the ERAS pathway in April 2017. The trial was conducted in accordance with the Declaration of Helsinki (as revised in 2013). The study was approved by the Local Ethics Committee (No. 2019-02464) and individual consent was waived.

\section{Data collection}

Data was retrospectively extracted from our electronic database. It included patient demographics, comorbidities, preoperative cardio-pulmonary functional testing, tumour stage and histology, operative characteristics, compliance to the ERAS protocol and postoperative outcomes, including overall and cardiopulmonary complications, duration of drainage and of hospital stay, readmissions and reoperations up to 30 days after surgery. The complications were defined as any adverse event influencing management, occurring within 30 postoperative days. Cardiopulmonary complications included: cardiac arrest, cardiac arrhythmia, acute myocardial ischemia, pneumothorax, hemothorax, prolonged air leak ( $\geq 7$ days), acute respiratory distress syndrome, pneumonia, acute pulmonary edema, massive subcutaneous emphysema, atelectasis, pulmonary embolism, chylothorax, empyema and bronchopulmonary fistula. The severity of complications was determined by the ClavienDindo classification, adapted for thoracic surgery, with grade I-II considered as minor and grade III-IV considered as major complications (18).

\section{ERAS program}

After 6 months of team education and training by a dedicated ERAS clinical nurse, surgeons and anaesthesiologists, an ERAS protocol for VATS anatomical pulmonary resection was finally launched in our thoracic surgery department in April 2017. Table 1 summarizes the differences of management before and after implementation of the ERAS program. This protocol was divided into three phases: preoperative, perioperative and postoperative.

\section{Preoperative phase}

Before surgery, all cases were discussed in an interdisciplinary thoracic board to review their oncological status and check their physiological tolerance to surgery. One or two weeks before surgery, the patients were seen by a multidisciplinary team in an outpatient clinic. The dedicated ERAS clinical 
Table 1 Internal guidelines for VATS lobectomy before (pre-ERAS) and after (ERAS) implementation of the ERAS program

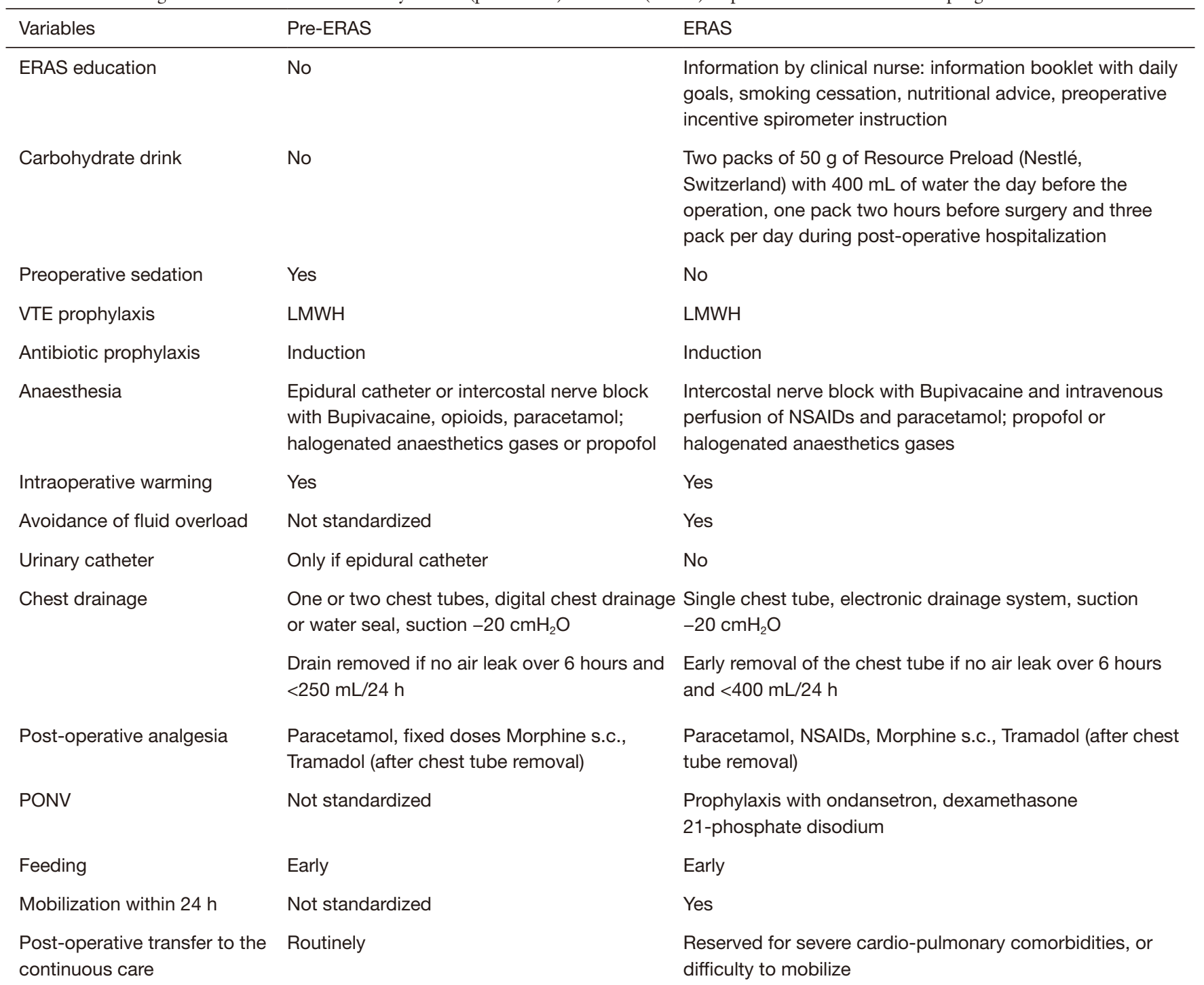

VTE, venous thromboembolism; LMWH, low-molecular-weight heparin; NSAIDs, nonsteroidal anti-inflammatory drugs; PONV, postoperative nausea and vomiting.

nurse informed the patient about the ERAS program concepts, the patient diary, the nutrition, the mobilization and the use of the incentive spirometer. The anaesthesiologist explained the analgesia and sedation procedures and emphasized the importance of smoking cessation before surgery. The surgeon explained the surgical steps and the expected post-operative outcomes and discharge procedure. The compliance was considered complete if the patient could meet all protagonists before surgery. The patients were admitted in our thoracic surgery department the day before the operation for logistical reasons, as is usual in our department. They received two packs of carbohydrate loading (50 g of Resource Preload ${ }^{\circledast}$ (Nestlé, Switzerland) with $400 \mathrm{~mL}$ of water) the day before the operation and one pack two hours before surgery.

\section{Perioperative phase}

No pre-anaesthesia sedative medication was given, except in case of important anxiety. Low-molecular-weight heparin (LMWH) was administered for venous thromboembolic (VTE) prophylaxis the day before surgery and once daily during the entire hospital stay. A weight-based dosage of 
cefuroxime was administered within $60 \mathrm{~min}$ prior to surgical incision as antibiotic prophylaxis. All patients underwent a VATS lobectomy under general anaesthesia with doublelumen tube and single-lung ventilation. The surgical technique consisted in a three ports anterior approach with individual dissection and transection of bronchovascular structures (19). Complete mediastinal lymph node dissection was carried out for all patients. After surgery, only one single chest tube was connected to an electronic drainage system (Thopaz, Medela ${ }^{\circ}$, Switzerland) with suction at $-20 \mathrm{cmH}_{2} \mathrm{O}$. In the majority of cases, the hypnotic used was Propofol by total intravenous anesthesia (TIVA) in order to reduce the likelihood of postoperative nausea and vomiting (PONV). The administration of volatiles was reserved for patients with moderate to severe chronic obstructive pulmonary disease (COPD) due to their bronchodilation properties. Before the end of the surgery, almost all patients received an intravenous perfusion of nonsteroidal anti-inflammatory drugs (NSAIDs; e.g., Ketorolac ${ }^{\circledR}$ ) and paracetamol. The administration of NSAIDs was avoided in case of renal impairment. Perioperative normothermia $\left(36-38^{\circ} \mathrm{C}\right)$ was achieved using forced air-warming blankets (Bair Hugger ${ }^{\mathrm{TM}}$ ). Fluid overload (defined as intra-operative hydration of $>1,000 \mathrm{~mL}$ of balanced fluid) and urinary drainage were avoided. Analgesia was controlled by a multimodal approach with an intercostal block and opioids. Epidural catheter was reserved for patients with high risk of conversion to thoracotomy (as opposed to pre-2017, when its use was more frequent) or in case of intolerance to opioids.

\section{Postoperative phase}

At the end of surgery, all patients were extubated and transferred to the ward when there were no associated cardiopulmonary comorbidities and sufficient autonomous mobilization. Otherwise, patients were admitted to the continuous care unit for surveillance during the first night. The PONV was prevented by a multimodal pharmacological therapy. Early feeding was provided by carbohydrate drinks and a meal given to the patients when they were totally recovered from anaesthesia. Intravenous fluids were stopped as soon as patients could drink by themselves. Daily early mobilization, in the form of two short walks in the ward with the help of a nurse, started on the day of the operation as soon as patients were fully awake, and was carried out twice daily on the following days until discharge. The chest tube was removed as soon as there was no air leak over 6 hours and the amount of pleural fluid was $<400 \mathrm{~mL}$ over 24 hours. Postoperative multimodal analgesia consisted in strong opioids (e.g., morphine), NSAIDs and paracetamol on post-operative day (POD) 0. Once the chest tube was removed, the strong opioids were replaced by oral weak opioids or derivatives (e.g., tramadol).

\section{Compliance}

The compliance to the ERAS protocol was based on the 16 individual items of the program described in Table 1, based on current evidence supporting their importance and general guidelines applicable to thoracic surgery. The compliance rate was defined as the number of protocol items observed divided by the total number of items.

\section{Statistical analysis}

Data was stratified by group (pre-ERAS vs. ERAS). Continuous variables were summarized as means [standard deviation (SD)] or medians [interquartile range (IQR)] and categorical variables as numbers (percentages). The Student $t$-test or Wilcoxon rank sum test was applied to compare the continuous variables between the groups, and the $\chi^{2}$ test or Fisher exact test was applied for comparing the categorical data.

To assess the impact of the ERAS protocol on length of hospital stay, readmission and complication rates within 30 days, we used a propensity-score matching (PSM). The PSM helped us to mitigate the selection bias regarding the distribution of the known covariates. The propensity score was calculated using a logistic regression model in which the protocol status is regressed against baseline covariates: age; sex; body-mass index (BMI); tumour localization (superior, middle or inferior lobe); comorbidities (high blood pressure, cardiopathy, arrhythmia, chronic obstructive pulmonary disease (COPD), tobacco exposure, diabetes, renal failure, immunosuppression and oncological history); ASA score; preoperative pulmonary functions ( $\mathrm{FEV}_{1}$ and DLCO). The fitted model did not include any interaction between covariates and assumed a linear relationship between continuous covariates and the log-odds of receiving the ERAS protocol. The analysis of the balancing property of the propensity score in the region of common support $(0.15,0.91)$ was performed and deemed satisfactory. Only one patient had no match in the region of common support and was thus excluded from the analysis, making a cohort of 306 patients (pre-ERAS: 166, ERAS: 140). The PSM was then used to estimate the average treatment effect (ATE) from the observed data. The PSM estimated the potential outcome for each individual by using an average of the outcomes of similar individuals that received the opposite 
protocol. There was similarity when the propensity-score of an individual and a match differ by less than $15 \%$ (caliper $=0.15$ ). The ATE was then calculated as the average of the difference between the observed and potential outcomes of each individual.

Data analysis was performed using Stata 16 Software (StataCorp. 2019. Stata Statistical Software: Release 16. College Station, TX: StataCorp LLC).

\section{Results}

In total, 307 patients with a median age of 67 years underwent VATS lobectomy for NSCLC (164 male/143 female; 167 pre-ERAS/140 ERAS). There was no substantial statistical difference in patient's characteristics between the groups regarding age, BMI, comorbidities, American Society of Anaesthesiologists (ASA) score, pre-operative pulmonary functions and tumour characteristics (Table 2). In both groups, most of the procedures involved the superior lobe (58.6\%), followed by the inferior lobe $(30 \%)$ and the middle lobe $(11.4 \%)$. More than $82 \%$ of all patients had an earlystage lung cancer (stage I-II). There was fewer stage I (55.7\% vs. $74.2 \% ; \mathrm{P}=0.0006)$ and more stage III $(18.6 \%$ vs. $7.2 \%$; $\mathrm{P}=0.0025)$ NSCLC in the ERAS group.

\section{Compliance to ERAS protocol}

After the introduction of our ERAS program, we observed a statistically significant increased rate of postoperative transfer to the ward $(46.3 \%$ vs. $8.4 \%$; $\mathrm{P}<0.00001)$, a decreased use of epidural catheter ( $9.3 \%$ vs. $29.3 \%$; $\mathrm{P}<0.00001)$ and a lower number of chest tube placement (mean $1.1 \pm 0.3$ vs. $1.4 \pm 0.5 ; \mathrm{P}<0.00001$ ). The overall compliance to the ERAS program was $81 \%$ (Figure 1).

\section{Unadjusted postoperative outcomes}

The unadjusted analysis of postoperative outcomes showed a shorter length of drainage (median 2 vs. 3 days; $\mathrm{P}=0.006$ ) and a shorter length of hospital stay (median 5 vs. 7 days; $\mathrm{P}=0.04$ ) in the ERAS group (Table 3). The overall 30-day morbidity rate was not statistically different between both groups ( $29.3 \%$ vs. $36.5 \%$; $\mathrm{P}=0.18)$. When analysing in details, there was also no difference in terms of cardiopulmonary $(27.1 \%$ vs. $35.9 \% ; \mathrm{P}=0.1)$ or severe $(9.3 \%$ vs. $10.8 \% ; \mathrm{P}=0.67$ ) complications (Clavien-Dindo III-IV). There was no 30-day mortality in the entire cohort. There was no statistical difference in terms of 30-day readmission rate $(3.6 \%$ vs. $5.4 \% ; \mathrm{P}=0.45)$ and necessity of re-drainage (5.7\% vs. 8.4\%; $\mathrm{P}=0.37$ ) between both groups.

\section{Propensity score}

The PSM analysis showed that the average length of hospital stay was significantly reduced by 1.4 days (95\% CI: -2.69 , $-0.11 ; \mathrm{P}=0.034)$ and the cardio-pulmonary complication rate was reduced by $13 \%$ (95\% CI: $-0.25,-0.003 ; \mathrm{P}=0.044)$ in the ERAS group (Table 3). For severe complications (ClavienDindo III-IV), the effect of the ERAS program was not statistically significant (ATE $=-0.05 ; 95 \%$ CI: $-0.11,0.05$; $\mathrm{P}=0.18$ ). Readmission (ATE $=-0.01 ; 95 \%$ CI: $-0.07,0.05$; $\mathrm{P}=0.75$ ) and re-drainage (ATE $=-0.05 ; 95 \% \mathrm{CI}:-0.12$, $0.006 ; \mathrm{P}=0.08)$ rates were similar between both groups. The duration of drainage was not statistically different between groups (ATE $=-0.82 ; 95 \% \mathrm{CI}:-1.9,0.26 ; \mathrm{P}=0.14$ ).

\section{Discussion}

We present here the postoperative outcomes of a large series of 307 patients undergoing VATS lobectomy for NSCLC before and after the implementation of a standardized ERAS protocol. After implementation of the ERAS program, we observed a decreased use of epidural catheter by $20 \%$, an increased rate of postoperative transfer to the ward by $38 \%$ and a decreased mean number of chest tube placement by 0.3 . The propensity score matched analysis shows that the length of hospital stay is significantly reduced, with a median shortening of 1.4 days $(\mathrm{P}=0.034)$ and the rate of postoperative cardiopulmonary complications significantly decreased by $13 \%(\mathrm{P}=0.044)$.

Since the introduction of the ERAS pathway in thoracic surgery and the recent publication of the international guidelines in 2019, several series have reported on the beneficial impacts of such a program (12-14,20,21). However, most of these series tend to aggregate various types of surgery (wedge, segmentectomy, lobectomy, pneumonectomy) and of surgical approaches (open, VATS, robotic). Therefore, the exact conclusions about the benefits of an ERAS pathway remain an open debate.

Indeed, each surgical approach and each type of surgery significantly influences the postoperative outcomes, with a known reduction of length of stay and postoperative complications in the patients undergoing minimally invasive procedures $(4,22)$. Thus, the introduction of an ERAS pathway may be expected to impact the outcome of VATS patients only moderately. In a retrospective series 
Table 2 Patient characteristics in the pre-ERAS and ERAS groups

\begin{tabular}{|c|c|c|c|}
\hline Variables & Pre-ERAS $(n=167)$ & ERAS $(n=140)$ & $P$ value \\
\hline Female & $69(41.3)$ & $74(52.9)$ & \\
\hline Male & $98(58.7)$ & $66(47.1)$ & \\
\hline Age (years), median (IQR) & $67(60$ to 74$)$ & 67 (59 to 72$)$ & 0.29 \\
\hline \multicolumn{4}{|l|}{ Comorbidities, n (\%) } \\
\hline High blood pressure & $82(49.1)$ & $69(49.3)$ & 0.97 \\
\hline Cardiopathy & $30(18.0)$ & $21(15.0)$ & 0.49 \\
\hline Arrhythmia & $30(18.0)$ & $13(9.3)$ & $0.03^{*}$ \\
\hline Diabetes & $29(17.4)$ & $17(12.1)$ & 0.2 \\
\hline Renal failure & $13(7.8)$ & $8(5.7)$ & 0.47 \\
\hline Immunosuppression & $3(1.8)$ & $4(2.9)$ & 0.54 \\
\hline ASA score, mean \pm SD & $2.5 \pm 0.5$ & $2.5 \pm 0.5$ & 0.59 \\
\hline \multicolumn{4}{|l|}{ Preoperative PFTs, mean \pm SD } \\
\hline FEV1 (\%) & $89.8 \pm 22.3$ & $85.8 \pm 20.3$ & 0.11 \\
\hline DLCO (\%) & $74.1 \pm 18.5$ & $72.6 \pm 20$ & 0.5 \\
\hline Neoadjuvant chemotherapy, n (\%) & $9(5.4)$ & $7(5)$ & 0.88 \\
\hline Stage I & $124(74.2)$ & $78(55.7)$ & $0.0006^{*}$ \\
\hline Stage II & $24(14.4)$ & $27(19.3)$ & 0.25 \\
\hline Stage III & $12(7.2)$ & $26(18.6)$ & $0.0025^{\star}$ \\
\hline Stage IV & $7(4.2)$ & $9(6.4)$ & 0.38 \\
\hline Adjuvant chemotherapy, n (\%) & $63(37.7)$ & $61(43.6)$ & 0.3 \\
\hline
\end{tabular}

*, $\mathrm{P}<0.05$. IQR, interquartile range; SD, standard deviation; BMI, body mass index; COPD, chronic obstructive pulmonary disease; ASA, American Society of Anaesthesiologists; PFTs, pulmonary function tests; FEV ${ }_{1}$, forced expiratory volume in one second; DLCO, diffusing capacity of the lung for carbon monoxide.

of 2,886 lung cancer resections, Van Haren et al. showed that the ERAS program was independently associated with a lower rate of pulmonary and cardiac complications after thoracotomy but not after VATS procedures.
Another retrospective series including a total of 363 patients (ERAS/non-ERAS: 139/224) found a statistically significant reduction of length of stay in ERAS patients after thoracotomy but not after VATS procedures (23). 


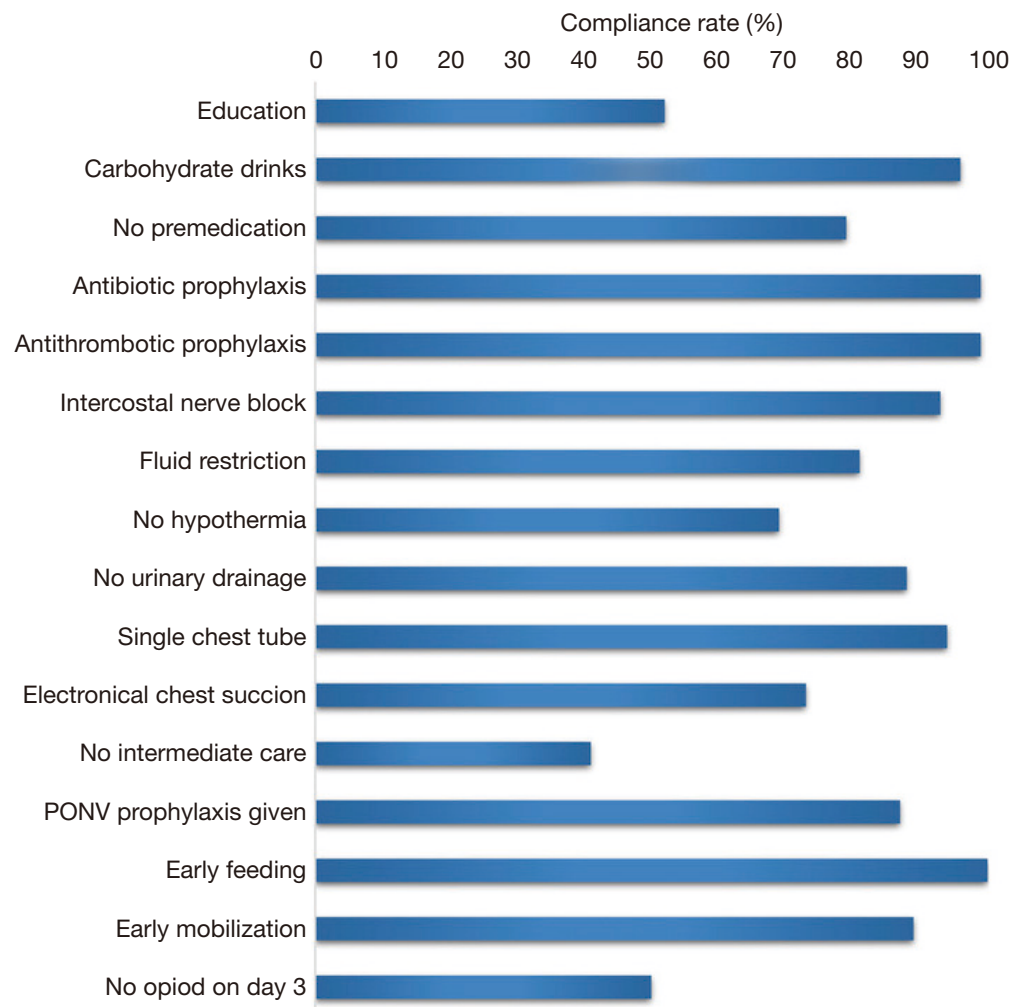

Figure 1 Compliance rate to individual elements of the ERAS program. PONV, post-operative nausea and vomiting.

Table 3 Unadjusted comparison of postoperative outcomes between the pre-ERAS and ERAS patients and estimated ATE in population using a propensity score-matched analysis

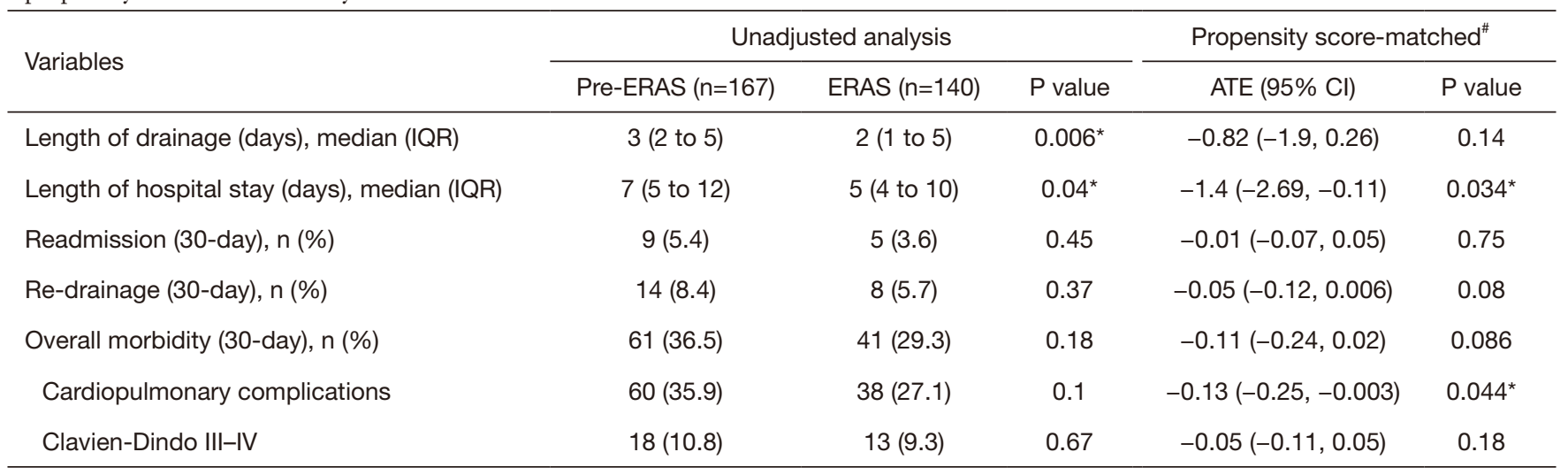

\#, propensity score based on: age; sex; body-mass index (BMI); tumour localization (superior, middle, or inferior lobe); comorbidities [high blood pressure, cardiopathy, arrhythmia, chronic obstructive pulmonary disease (COPD), tobacco exposure, diabetes, renal failure, immunosuppression, and oncological history]; ${ }^{*}, \mathrm{P}<0.05$. ASA score; preoperative pulmonary functions $\left(\mathrm{FEV}{ }_{1}\right.$ and $\left.\mathrm{DLCO}\right)$. $\mathrm{Cl}$, confidence interval; ATE, average treatment effect. 
On the contrary, Haro et al. did not find any influence of the surgical approach (open $v s$. minimally invasive) on the reduction of postoperative morbidity, length of stay and costs in ERAS patients in their propensity score-matched study (14). However, the extent of pulmonary resection (wedge, segmentectomy, lobectomy, pneumonectomy) and/or the pathology (primary lung cancer, pulmonary metastasis, benign lesion) were not considered in these studies. A more selective study including only patients undergoing lobectomy or segmentectomy by VATS did not report any statistical difference in terms of length of stay or cardiopulmonary complications between the ERAS and non-ERAS patients (24). These results were mainly explained by the fact that the introduction of the ERAS program did not translate into major differences from their prior routine practice.

In our department, the introduction of an ERAS pathway for VATS anatomical resections changed our routine practices and showed beneficial results. We previously reported on a series of 100 patients (ERAS/non-ERAS: 50/50) with lung cancer undergoing VATS segmentectomy or lobectomy (13). This series showed significantly shorter postoperative length of stay (median $4 v s .7$ days; $\mathrm{P}<0.0001$ ), lower rate of overall postoperative complications $(24 \%$ vs. $48 \%$; $\mathrm{P}=0.03$ ) and lower total hospitalization costs $(€ 15,945$ vs. $€ 20,360$; $\mathrm{P}<0.0001)$ in the ERAS group. However, the groups were not fully matched with better preoperative pulmonary functions and lower ASA score in the ERAS group. To avoid these potential biases, we decided to compare two groups of VATS lobectomy patients in a propensity score-matched analysis to see if the benefit of the ERAS program was real. For obvious reasons, the population of patients included in this study can be divided by the time of their inclusion: before April 2017, all patients were in the "pre-ERAS" group, and after that time, in the "ERAS" group. This could have been a source of a time-related selection bias, as it would have included the ERAS learning curve. On the other hand, if that had been the case, this would have mitigated our results towards smaller differences. Thus, the fact that we can draw conclusions shows that even the learning curve period was not detrimental enough that the benefits we report would vanish. Yet, the main observation is this one: the team involved in the study, including the surgeons and all the surgical team had a wide expertise in this type of operation (started in 2010), even before the ERAS program was introduced in our institution. Similarly, the techniques we used did not significantly change since April 2017. By way of consequence, this consistency in team and techniques allowed us to smooth out any time- related selection bias. Thus, by the time the ERAS program was rolled in, each member of the surgical team already had a good experience on VATS lobectomy and honed the necessary technical skills.

In the present study, the postoperative cardiopulmonary complication rate was lower in the ERAS group than in the pre-ERAS group ( $27.1 \%$ vs. $35.9 \%)$, not reaching statistically significant in the non-adjusted analysis. in the propensity score-matching analysis, the result became statistically significant and clinically meaningful, with a reduction of cardiopulmonary complication rate of $13 \%$ $(\mathrm{P}=0.044)$, which is in agreement with other series $(13,15)$. In their meta-analysis, Li et al. showed a significant reduction in pulmonary and surgical complications in the ERAS group (15). It is to be noted that in our series, the rate of severe complications (Clavien-Dindo III-IV) was similar between both groups $(\mathrm{P}=0.18)$. Following the reduction of postoperative complications, patients' recovery is accelerated and length of hospital stay is reduced (13$17,23,25)$. A previous propensity score-matched study including 295 lung cancer patients undergoing various pulmonary resections showed a significant reduction of length of hospital stay of 1.2 days in the ERAS group (14), which is similar to our result $(-1.4$ days; $\mathrm{P}=0.034)$. Interestingly, our length of drainage and readmission rates were not statistically different between the pre-ERAS and ERAS patients, as demonstrated by other groups $(16,17,25)$. As the length of drainage is generally short and the readmission rate low in this specific patients' population in our institution, even before the introduction of an ERAS program, a change would be difficult to measure. Finally, we also observe that in a recent study, Tahiri et al. report a reduction of post-operative length of stay in a cohort of patients who underwent VATS lobectomy and were managed within the scope of an ERAS protocol, as compared to pre-ERAS care (26), results that are very much in keeping with our own. In more general terms, we note that this recent study is of similar nature to ours insofar as it avoids some of the pitfalls that we wished to avoid in designing our study: most studies tend to aggregate various types of surgical approaches and operations as they include wedge resections and thoracotomy procedures. The study by Tahiri et al. and ours take the reasoning one step further by exclusively reporting on VATS lobectomies for NSCLCs. The results of both studies are in keeping. This is of much scientific relevance since the settings are quite different for the two studies (Canada and Europe; differential sizes of patient populations; different periods 
of time and study durations; developing ERAS pathway vs. established protocol; different compliance rates).

A key element of the ERAS program is the compliance of patients. Indeed, the benefits of the program correlate to the level of protocol compliance. Our previous series of 192 ERAS patients undergoing VATS anatomical pulmonary resection showed that a high compliance to the ERAS protocol $(\geq 75 \%)$ was associated with a lower rate of postoperative complications and delayed discharge (20). Another study including 422 patients confirmed a significant inverse correlation between protocol compliance and postoperative morbidity (27). However, a complete adherence to the ERAS protocol is difficult. Many barriers have been described in literature, such as the lack of institutional support, the resistance to change, the poor communication and collaboration between team members and the patient variability $(28,29)$. In our study, the overall compliance rate to the ERAS protocol was $81 \%$. During the introduction of our protocol, we experienced several difficulties, such as the organization of the systematic outpatient preoperative consultation. Even if all patients were seen by the clinical nurse in hospital the day before surgery, we considered that a preoperative outpatient consultation was necessary in order to provide detailed information and education about the ERAS program. Thereby, patients may feel more involved in their management and their subsequent compliance, potentially translated in a higher satisfaction. Regarding the team collaboration, we continuously reviewed the compliance to the ERAS items as part of an internal audit process taking place every three months. This allowed a progressive harmonization of practices among all caregivers. Nowadays, the ERAS protocol for VATS anatomical pulmonary resection is well established in our department and has also shown additional organizational benefits. The standardization of the patient management has greatly facilitated the work of young doctors and their supervision. In addition, the implication of the nurses for the patient's mobilization and the higher rate of patients transfer to the ward indirectly benefited patients undergoing other surgical procedures like thoracotomy, for which a protocol has been developed in parallel.

Early mobilization has been reported to be a central element of the ERAS pathway (ERAS/ESTS guidelines) to avoid the deleterious effects of bed rest. Rogers et al. reported that early mobilization was a strong predictor of decreased postoperative complications and shortened length of stay, but this study was unclear about which type of mobilization (27). Mazza et al. reported a shortened length of stay in their ERAS group patients when patients were mobilized into a sitting position within 4 hours from tracheal tube weaning and when physiotherapy started within 12 hours after surgery (30). The series reported by Khendaar et al. reported an aggressive regimen of mobilization with VATS lobectomy patients placed on a chair as soon as possible with an ambulation target of 250 feet within one hour of extubation. A majority of patients $(61.5 \%)$ achieved this ambulation goal (31). In our protocol, $89 \%$ of patients were mobilized on day of surgery. They were seated for dinner and walked through the ward helped by nurses from the first day onwards.

By accelerating recovery, ERAS programs may also facilitate access and tolerance to adjuvant chemotherapy for NSCLC. Recently, Nelson et al. investigated 471 patients treated for NSCLC by either open or VATS approach. They observed a shortened interval between lung resection and beginning of adjuvant chemotherapy. The rate of adjuvant chemotherapy also increased progressively from $40 \%$ during the pre-ERAS phase to $50 \%$ during the transition era, and finally $62 \%$ during the ERAS era $(\mathrm{P}<0.001)(32)$. In our study, the rate of adjuvant chemotherapy was slightly higher in the ERAS group ( $43.6 \%$ vs. $37.7 \%$ ), but the difference was not statistically significant $(\mathrm{P}=0.3)$.

The main limitation of this study is the retrospective, single centre design. However, our institution captures most cases from the French speaking part of Switzerland, thus representing a large national population, which lowers the selection bias. Another limitation is the differential distribution of patients over time between the two groups. Indeed, the ERAS program was introduced in our institution in April 2017. Thereby, the first part of the cohort included only pre-ERAS patients and the later part of the cohort represented only ERAS patients. However, the involved surgeons had a wide expertise in VATS lobectomy procedures before the start of the study and the surgical teams and techniques did not significantly change during the study period.

We also observed a difference in the populations involved in the study groups: we found fewer cases of stage I (and more advanced stage NSCLCs) in the ERAS group. Whilst this may be the cause or the consequence of a bias, we do not have a clear explanation since the rate of neoadjuvant chemotherapy were similar in both groups, but we know that we did not modify the selection criteria for lung cancer surgery during the study period or extend indication for multimodal treatments, thus excluding a direct selection bias. One possible explanation is that we may have operated larger lesions with growing experience and thus observed 
more incidental N1-2. Interestingly, even with such difficult cases, we reported better post-operative outcomes.

\section{Conclusions}

In conclusion, in our experience, the introduction of an ERAS pathway for VATS lobectomy in lung cancer patients has resulted in a shortened length of hospital stay and decreased rate of postoperative cardiopulmonary complications without affecting the rate of readmissions.

\section{Acknowledgments}

Funding: None.

\section{Footnote}

Reporting Checklist: The authors have completed the STROEB reporting checklist. Available at http://dx.doi. org/10.21037/tlcr-20-891

Data Sharing Statement: Available at http://dx.doi. org/10.21037/tlcr-20-891

Peer Review File: Available at http://dx.doi.org/10.21037/ tlcr-20-891

Conflicts of Interest: All authors have completed the ICMJE uniform disclosure form (available at http://dx.doi. org/10.21037/tlcr-20-891). The authors have no conflicts of interest to declare.

Ethical Statement: The authors are accountable for all aspects of the work in ensuring that questions related to the accuracy or integrity of any part of the work are appropriately investigated and resolved. The trial was conducted in accordance with the Declaration of Helsinki (as revised in 2013). The study was approved by the Local Ethics Committee (No. 2019-02464) and individual consent was waived.

Open Access Statement: This is an Open Access article distributed in accordance with the Creative Commons Attribution-NonCommercial-NoDerivs 4.0 International License (CC BY-NC-ND 4.0), which permits the noncommercial replication and distribution of the article with the strict proviso that no changes or edits are made and the original work is properly cited (including links to both the formal publication through the relevant DOI and the license). See: https://creativecommons.org/licenses/by-nc-nd/4.0/.

\section{References}

1. Global Burden of Disease Cancer Collaboration, Fitzmaurice C, Dicker D, et al. The Global Burden of Cancer 2013. JAMA Oncol 2015;1:505-27.

2. Howington JA, Blum MG, Chang AC, et al. Treatment of stage I and II non-small cell lung cancer - Diagnosis and management of lung cancer, 3rd ed: American college of chest physicians evidence-based clinical practice guidelines. Chest 2013;143:e278S-313S.

3. Whitson BA, Groth SS, Duval SJ, et al. Surgery for earlystage non-small cell lung cancer: a systematic review of the Video-Assisted Thoracoscopic Surgery versus thoracotomy approaches to lobectomy. Ann Thorac Surg 2008;86:200816; discussion 2016-8.

4. Falcoz PE, Puyraveau M, Thomas PA, et al. Video-assisted thoracoscopic surgery versus open lobectomy for primary non-small-cell lung cancer: a propensity-matched analysis of outcome from the European Society of Thoracic Surgeon database. Eur J Cardiothorac Surg 2016;49:602-9.

5. Boffa DJ, Dhamija A, Kosinski AS, et al. Fewer complications result from a video-assisted approach to anatomic resection of clinical stage I lung cancer. J Thorac Cardiovasc Surg 2014;148:637-43.

6. Laursen LØ, Petersen RH, Hansen HJ, et al. Videoassisted thoracoscopic surgery lobectomy for lung cancer is associated with a lower 30-day morbidity compared with lobectomy by thoracotomy. Eur J Cardiothorac Surg 2016;49:870-5.

7. Gustafsson UO, Scott MJ, Schwenk W, et al. Guidelines for perioperative care in elective colonic surgery: Enhanced Recovery After Surgery (ERAS) society recommendations. World J Surg 2013;37:259-84.

8. Spanjersberg WR, Reurings J, Keus F, et al. Fast track surgery versus conventional recovery strategies for colorectal surgery (Review). Cochrane Database Syst Rev 2011;(2):CD007635.

9. Zhuang CL, Ye XZ, Zhang XD, et al. Enhanced recovery after surgery programs versus traditional care for colorectal surgery: a meta-analysis of randomized controlled trials. Dis Colon Rectum 2013;56:667-78.

10. Mortensen K, Nilsson M, Slim K, et al. Consensus guidelines for enhanced recovery after gastrectomy - Enhanced Recovery After Surgery (ERAS) Society recommendations. Br J Surg 2014;101:1209-29. 
11. Nelson G, Bakkum-Gamez J, Kalogera E, et al. Guidelines for perioperative care in gynecologic/oncology: Enhanced Recovery After Surgery (ERAS) Society recommendations - 2019 update. Int J Gynecol Cancer 2019;29:651-68.

12. Batchelor TJP, Rasburn NJ, Abdelnour-Berchtold E, et al. Guidelines for enhanced recovery after lung surgery: recommendations of the Enhanced Recovery After Surgery (ERAS) Society and the European Society of Thoracic Surgeons (ESTS). Eur J Cardiothorac Surg 2019;55:91-115.

13. Gonzalez M, Abdelnour-Berchtold E, Perentes JY, et al. An enhanced recovery after surgery program for videoassisted thoracoscopic surgery anatomical lung resections is cost-effective. J Thorac Dis 2018;10:5879-88.

14. Haro GJ, Sheu B, Marcus SG, et al. Perioperative lung resection outcomes after implementation of a multidisciplinary, evidence-based thoracic ERAS program. Ann Surg 2019. [Epub ahead of print]. doi: 10.1097/ SLA.0000000000003719

15. Li S, Zhou K, Che G, et al. Enhanced recovery programs in lung cancer surgery: systematic review and metaanalysis of randomized controlled trials. Cancer Manag Res 2017;9:657-70.

16. Madani A, Fiore JF, Wang Y, et al. An enhanced recovery pathway reduces duration of stay and complications after open pulmonary lobectomy. Surgery 2015;158:899-908.

17. Paci P, Madani A, Lee L, et al. Economic impact of an enhanced recovery pathway for lung resection. Ann Thorac Surg 2017;104:950-7.

18. Dindo D, Demartines N, Clavien PA. Classification of surgical complications - A new proposal with evaluation in a cohort of 6336 patients and results of a survey. Ann Surg 2004;240:205-13.

19. Hansen HJ, Petersen RH, Christensen M. Videoassisted thoracoscopic surgery (VATS) lobectomy using a standardized anterior approach. Surg Endosc 2011;25:1263-9.

20. Forster C, Doucet V, Perentes JY, et al. Impact of compliance with components of an ERAS pathway on the outcomes of anatomic VATS pulmonary resections. J Cardiothorac Vasc Anesth 2020;34:1858-66.

21. Comacchio GM, Monaci N, Verderi E, et al. Enhanced recovery after elective surgery for lung cancer patients: analysis of current pathways and perspectives. J Thorac Dis 2019;11:S515-22.

22. Paul S, Altorki NK, Sheng S, et al. Thoracoscopic lobectomy is associated with lower morbidity than open lobectomy: A propensity-matched analysis from the STS database. J Thorac Cardiovasc Surg 2010;139:366-78.
23. Martin LW, Sarosiek BM, Harrison MA, et al. Implementing a thoracic enhanced recovery program: lessons learned in the first year. Ann Thorac Surg 2018;105:1597-604.

24. Brunelli A, Thomas C, Dinesh P, et al. Enhanced recovery pathway versus standard care in patients undergoing videoassisted thoracoscopic lobectomy. J Thorac Cardiovasc Surg 2017;154:2084-90.

25. Van Haren RM, Mehran RJ, Mena GE, et al. Enhanced recovery decreases pulmonary and cardiac complications after thoracotomy for lung cancer. Ann Thorac Surg 2018;106:272-9.

26. Tahiri M, Goudie E, Jouquan A, et al. Enhanced recovery after video-assisted thoracoscopic surgery lobectomy: a prospective, historically controlled, propensity-matched clinical study. Can J Surg 2020;63:E233-40.

27. Rogers LJ, Bleetman D, Messenger DE, et al. The impact of enhanced recovery after surgery (ERAS) protocol compliance on morbidity from resection for primary lung cancer. J Thorac Cardiovasc Surg 2018;155:1843-52.

28. Pearsall EA, Meghji Z, Pitzul KB, et al. A qualitative study to understand the barriers and enablers in implementing an Enhanced Recovery After Surgery Program. Ann Surg 2015;261:92-6.

29. Springer JE, Doumouras AG, Lethbridge S, et al. A provincial assessment of the barriers and utilization of Enhanced Recovery After Colorectal Surgery. J Surg Res 2019;235:521-8.

30. Mazza F, Venturino M, Turello D, et al. Enhanced recovery after surgery: adherence and outcomes in elderly patients undergoing VATS lobectomy. Gen Thorac Cardiovasc Surg 2020;68:1003-10.

31. Khandhar SJ, Schatz CL, Collins DT, et al. Thoracic enhanced recovery with ambulation after surgery: a 6-year experience. Eur J Cardiothorac Surg 2018;53:1192-8.

32. Nelson DB, Mehran RJ, Mitchell KG, et al. Enhanced recovery after thoracic surgery is associated with improved adjuvant chemotherapy completion for nonsmall cell lung cancer. J Thorac Cardiovasc Surg 2019;158:279-86.e1.

Cite this article as: Forster C, Doucet V, Perentes JY, Abdelnour-Berchtold E, Zellweger M, Faouzi M, Bouchaab H, Peters S, Marcucci C, Krueger T, Rosner L, Gonzalez M. Impact of an enhanced recovery after surgery pathway on thoracoscopic lobectomy outcomes in non-small cell lung cancer patients: a propensity score-matched study. Transl Lung Cancer Res 2021;10(1):93-103. doi: 10.21037/tlcr-20-891 